\title{
Developing a PLC-friendly state machine model: lessons learned
}

\author{
Wim Pessemier ${ }^{a}$, Geert Deconinck ${ }^{b}$, Gert Raskin $^{a}$, Philippe Saey ${ }^{c}$ and Hans Van Winckel ${ }^{a}$ \\ ${ }^{a}$ Institute of Astronomy, KU Leuven, Leuven, Belgium; \\ ${ }^{b}$ ESAT - dept. of Electrical Engineering, KU Leuven, Leuven, Belgium; \\ ${ }^{c} \mathrm{E} \& \mathrm{~A}$ - Electrical Engineering, FET, KU Leuven - campus Gent, Gent, Belgium
}

\begin{abstract}
Modern Programmable Logic Controllers (PLCs) have become an attractive platform for controlling real-time aspects of astronomical telescopes and instruments due to their increased versatility, performance and standardization. Likewise, vendor-neutral middleware technologies such as OPC Unified Architecture (OPC UA) have recently demonstrated that they can greatly facilitate the integration of these industrial platforms into the overall control system. Many practical questions arise, however, when building multi-tiered control systems that consist of PLCs for low level control, and conventional software and platforms for higher level control. How should the PLC software be structured, so that it can rely on well known programming paradigms on the one hand, and be mapped to a well organized OPC UA interface on the other hand? Which programming languages of the IEC 61131-3 standard closely match the problem domains of the abstraction levels within this structure? How can the recent additions to the standard (such as the support for namespaces and object-oriented extensions) facilitate a model based development approach? To what degree can our applications already take advantage of the more advanced parts of the OPC UA standard, such as the high expressiveness of the semantic modeling language that it defines, or the support for events, aggregation of data, automatic discovery, ... ? What are the timing and concurrency problems to be expected for the higher level tiers of the control system due to the cyclic execution of control and communication tasks by the PLCs? We try to answer these questions by demonstrating a semantic state machine model that can readily be implemented using IEC 61131 and OPC UA. One that does not aim to capture all possible states of a system, but rather one that attempts to organize the course-grained structure and behaviour of a system. In this paper we focus on the intricacies of this seemingly simple task, and on the lessons that we've learned during the development process of such a "PLC-friendly" state machine model.
\end{abstract}

Keywords: state machine, semantic modeling, PLC, OPC UA

\section{INTRODUCTION}

Over the last few decades, innovations in information technology have been re-shaping the landscape of industrial automation systems significantly. Control systems have become more distributed, better integrated within enterprise systems, can meet higher real-time demands, and have become more "open" as the suppliers converge to programming and communication standards. In certain applications, the role of the PLC as a simple "programmable logic controller" is now being taken over by so-called "PC-based" controllers, offering a more versatile platform at the cost of increased complexity. This versatility is what makes the latter technology so attractive: real-time control software can be written in more languages (not only standard IEC 61131-3 but also $\mathrm{C}++$ or Matlab/Simulink models for instance), the embedded operating system can run more applications (such as communication servers, user interfaces, databases, ...), and one can take advantage of more recent hardware innovations (such as multi-core architectures).

At the same time, it seems that information technology advancements will continue to push the boundaries of conventional automation even further. Efforts such as Industrie 4.0 promise the dawn of "smart" factories, consisting of cyber-physical systems that collaboratively control and optimize manufacturing processes in a flexible way. ${ }^{1}$ The core ideas of Industrie 4.0 are all about integration, and they can be applied easily to astronomical telescope facilities:

wim.pessemier@ster.kuleuven.be 
- end-to-end integration: an integrated development approach that encompasses several domains may enable astronomers and engineers to deal with the complexity of future Extremely Large Telescopes;

- vertical integration: a departure from the traditional "three layered architecture" to a vertically integrated architecture from the field level up to the facility level makes reconfiguration of the telescope and instruments more flexible;

- horizontal integration: can assist decentralized control within a single facility (e.g. the telescope optics coordinating with an instrument) or even across observatory boundaries (e.g. two telescopes collaborating to optimize the available observation time).

Recent technologies such as PC-based controllers and OPC Unified Architecture play an important role in the realization of the above ideas, because they narrow the gap between the traditional automation world and the IT-world. ${ }^{2}$ Semantic modeling (or ontology engineering) is an additional discipline that can help to achieve these goals. Applied to industrial applications, ontologies can uniquely identify (and organize, and even formally define) all relevant parts and properties of a system, and link them together regardless of the engineering discipline they belong to. This holistic idea of "semantic integration" is one way to achieve a tighter horizontal/vertical/end-toend integration as advocated by Industrie 4.0. Furthermore, because the semantics of the systems are formally specified, it is possible to add logic or rules to the models in such a way that conclusions (or "inferences") can be drawn by software algorithms. ${ }^{3}$ This reasoning capability is what makes semantic applications "smart".

At the Mercator telescope, we already developed a software infrastructure that can accommodate semantic models of our instrumentation. In this paper however, we will focus on a particular ontology that we have developed within our framework: one that allows us to model the states and behaviour of mechatronic systems. We will show the technologies and tools that were used to develop the ontology, and how it can be used to model control systems. We will then focus on how the state machine models can be converted into PLC software code, the pitfalls we had to avoid, and the lessons we've learned throughout the development process.

\section{A SEMANTIC STATE MACHINE MODEL}

\subsection{Ontology development}

In a minimal sense, an ontology can be considered a vocabulary: a list of "things" within a given domain, identified by a URI (Unified Resource Identifier). In case of our state machine domain, we have listed concepts (such as State and Transition) and predicates (such as hasState and isTransitionFrom), and added them to an ontology identified by the URI http://mercator.iac.es/onto/metamodels/finitestatemachines. To reduce the burden of this long URI, we assigned a prefix (fsm) to the ontology. In the rest of this paper we will explicitly include this and other prefixes to relate all "things" to a particular ontology. The prefixes are essentially qualifiers: they avoid the disambiguity that can occur between for instance fsm:State and place:State (the latter being defined as "a land area of governance within a country" by the Places Ontology ${ }^{4}$ ).

Ontologies usually do more than vocabularies: they can be considered taxonomies as they organize all things into hierarchical groups. We use the semantics of Semantic $\mathrm{Web}^{5}$ languages $\mathrm{RDF}(\mathrm{S})$ and $\mathrm{OWL}^{*}$ to organize our fsm ontology. For instance, we specified that all our concepts are a $r d f s$ :subClass0f the owl:Thing concept. A $f_{s m}$ :Condition on the other hand is a $r d f s:$ subClass0f the expr:BooleanExpression concept (the latter representing a boolean expression by our http://mercator.iac.es/onto/metamodels/expressions ontology).

Finally, ontologies can capture much more knowledge about things. Just as a dictionary defines the meaning (semantics) of words by relating them to other words, an ontology can formally define concepts by relating them to other concepts. An orthogonal region for instance can be formalized as "something that has at exactly one active state". This definition can be expressed in OWL as shown in the owl:equivalentClass field of Fig. 1. The same figure also shows how we have added a further restriction to the definition of our $f$ sm:Region concept: all states of a region must depend on the same variables. Even more knowledge is hidden behind the scenes: for instance "fsm:hasState" is defined as a relation that always relates an orthogonal region with a state. So

${ }^{*}$ The Resource Description Framework (Schema) RDF(S) and the Web Ontology Language OWL are the main formal languages to construct new ontologies for the Semantic Web. 
whenever we specify " $\mathbf{x} f s m$ :hasState $\mathbf{y}$ ", a smart application will infer that $\mathbf{x}$ is an instance of the $f_{s m}$ :Region concept, and $\mathbf{y}$ is an instance of the $f_{s m}$ :State concept. So-called heavy-weight ontologies can go even further by specifying rules that can be executed by an inference engine. Our fsm ontology contains for instance the rule that "if a state has a boolean condition consisting of variables, then the state depends on those variables". Rules like this are very hard or even impossible to express using OWL, but recent query-based rule languages such as SPIN (the SPARQL Inferencing Notation ${ }^{6}$ ) offer a convenient solution in those situations.

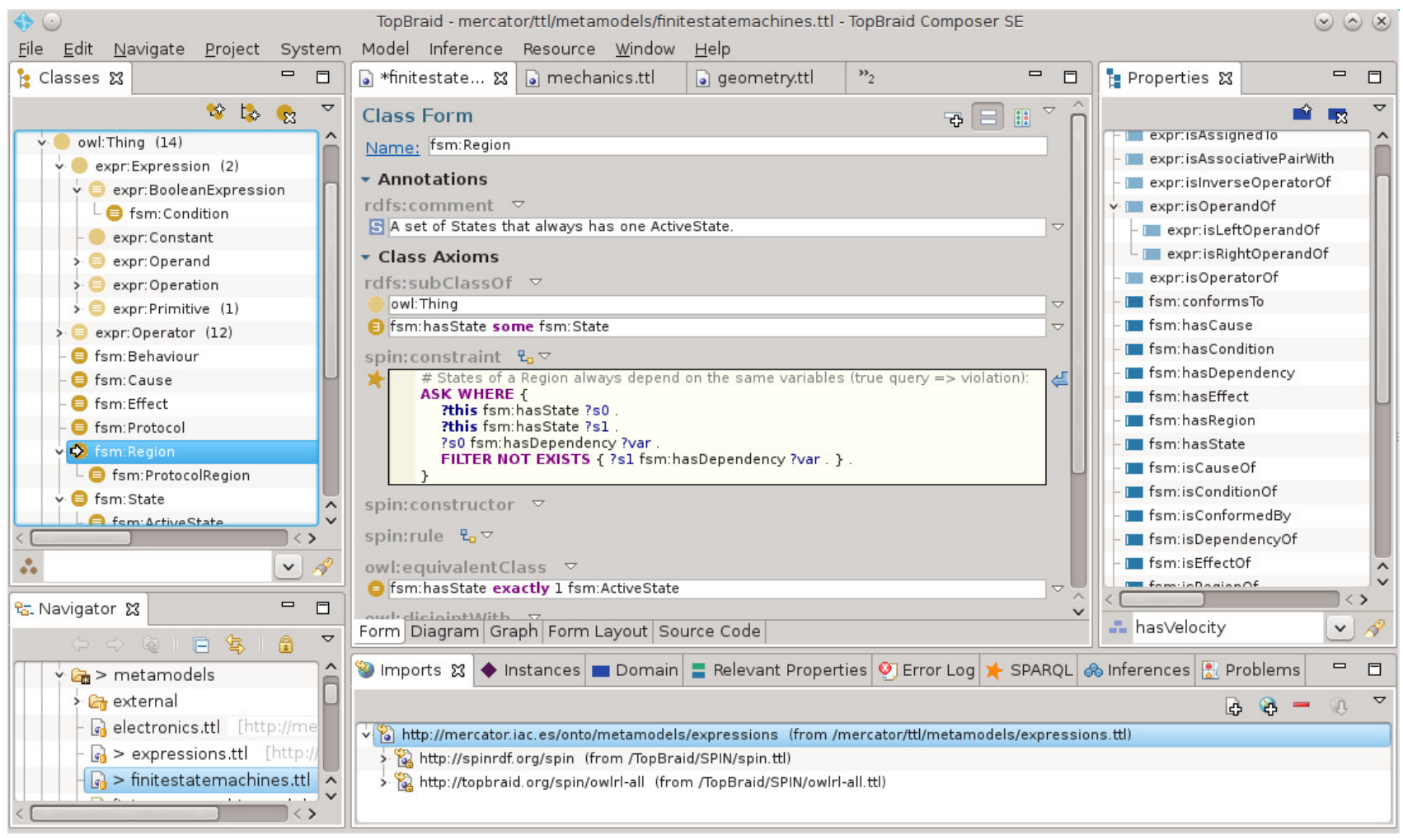

Figure 1. Developing the fsm ontology.

\subsection{Modeling basic state machines}

Below, we list the basic concepts of our finite state machine ontology. For each concept, we mention both the qualified name and a short summary of its formal definition.

- $f_{s m}$ :Condition: a expr:BooleanExpression related to a $f_{s m}$ :State.

- $f_{s m}$ :State: something that has a $f_{s m}$ :Condition.

- $f_{s m}$ StateVariable: a dependency of a $f_{s m}$ State.

- $f s m:$ ActiveState: a $f s m:$ State which condition is asserted to be true.

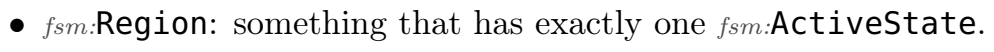

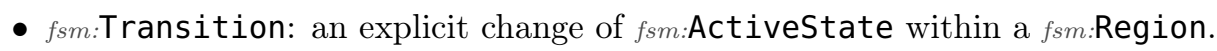

- $f_{s m}:$ StateMachine: a set of $f_{s m}$ :Regions.

- $f_{s m}$ :ComplexState: a $f_{s m}$ :State that is at the same time also a $f_{s m}$ :StateMachine. 
- $f_{s m}:$ ProtocolstateMachine: a kind of $f_{s m}:$ StateMachine to which other $f_{s m}:$ StateMachines can conform.

- $f_{s m}$ :ProtocolRegion: a kind of $f_{s m}:$ Region to which other $f_{s m}$ :Regions can conform.

An important remark is that Semantic Web technologies embrace the "Open World Assumption" or OWA. This assumption says that any observation may be true unless it is explicitly stated to be false. Applied to our use case, it means that we can only interpret the behaviour of a state machine by its known states and known transitions. Consider for instance a state machine with two states called A and B, and a transition from A to B. Based on this information, we cannot simply conclude that there is no transition from B to A. Only if we would add the statement "B cannot transit to A" to our knowledge base, then we can infer that an active state change from B to A would be invalid. In practice, this means that our state machine models do not have to be extensive and "complete" descriptions: only the relevant knowledge must be modeled. More states and more transitions can always be added later, without invalidating the original state machine model.

Inspired by the UML 2 (Unified Modeling Language version 2) concept of "protocol state machines", we have defined the concepts of $f_{s m}$ :ProtocolRegion and $f_{s m}$ :ProtocolstateMachine, as subclasses (according to RDF-S semantics) of $f_{s m}$ :Region and $f_{s m}$ :StateMachine respectively. Instances of these concepts do not exhibit behaviour, but instead they represent "abstract" regions and state machines that specify states and conditions w.r.t. unspecified state variables. Non-protocol regions and state machines can be said to "conform" to their counterpart via the $f_{s m}$ :conformsTo relation, which means that their states (and state variables and conditions) correspond one-to-one to the protocol. The reason for defining protocol regions and state machines is re-usability: all protocol definitions in the semantic model can easily be mapped to a software type (e.g. a Python class or an IEC 61131-3 function block).

\subsection{Modeling behaviour}

The vocabulary listed above allows us to identify some (but not necessarily all) states of a system, and to reason about how these states are affected by changes of variables that are somehow linked to the system. To model the actual mechanisms that drive these changes, we need additional syntax. Languages such as UML and SysML (the Systems Modeling Language) for instance provide "activity" diagrams to model processes and low-level behaviour. In our experience though, the added value of capturing low-level behaviour with this kind of diagrams is often very limited. Instead, it is often much easier to capture the low-level behaviour directly in a general purpose programming language, or a domain specific modeling tool such as Matlab/Simulink.

On the other hand, we feel that it does make sense to capture high-level behaviour with a semantic model. Consider a brushless DC-servomotor. We know that when this motor is running above its maximum recommended velocity, its temperature may rise to unacceptable levels. Instead of modeling the full thermal behaviour of the motor (which is better done by a numeric simulation tool), we can simply define a cause (i.e. the motor is running at overspeed) and link it to an effect (i.e. the motor is in overtemperature) that will take place in a given time span (e.g. 2 minutes). ${ }^{\dagger}$ The only concepts needed to describe this knowledge are:

- $f s m:$ Behaviour: something that has a $f s m: C a u s e$ and an $f s m:$ Effect, and optionally some time constraints.

- $f_{s m}$ :Cause: an expr:Expression, a $f_{s m}$ State or a $f_{s m}$ :Transition that is causing some $f_{s m}$ Behaviour.

- $f_{s m}$ :Effect: an expr:Expression, a $f s m:$ State or a $f s m:$ Transition that is the result of some $f_{s m}$ :Behaviour.

In many situations, this simple "if-cause-then-effect" paradigm is useful to apply to our modeled systems. We identify the following use cases:

\footnotetext{
${ }^{\dagger}$ In reality, many more causes (such as the motor load, the cooling provisions, the duty cycle, the safety factor used by the manufacturer, ...) will influence the thermal behaviour of the particular motor. However, since we didn't include those causes in our model, they don't participate in the modeled behaviour. Just like any other model, our semantic model captures our interpretation of reality.
} 


\section{System analysis.}

Consider the example of the electric motor running in overtemperature. Not only overspeeding may lead to unacceptable temperatures, but also an engaged brake may have the same effect. The brake at its turn may only be engaged (an effect) when the safety circuit is opened (a cause) or the power is lost (another cause). This kind of causes and effects are very easy to represent by our ontology. The advantage is that they can be analyzed, e.g. for troubleshooting purposes. A single query can answer a question such as "What are all the known causes (even recursively) of a dangerous temperature of motor X?" or "What are all the known effects (even recursively) of opening the safety circuit?".

\section{Code generation.}

When the semantic state machine model represents a piece of software, causes and effects can easily be mapped to an if-then construct by most software languages. As will be explained in the next section, we have developed ontologies that can map state machines (including physical objects such as a motor) to software models. Not only the states, regions, conditions etc. can be mapped to the software models, but also the known behaviour. For instance, the software representation of our motor state machine can implement the "overtemperature" behaviour as an if-then construct. As a result, the software representation can generate a warning when it has detected an overspeed for at least two minutes.

\section{Verification and validation of requirements.}

Some of the behaviour of our semantic models are essentially requirements. For instance, we can model the following requirement for the safety circuit: "if the safety circuit is opened, then the motor should be standing still within 1 second". This requirement can be modeled by a cause, an effect, and a time constraint. Using the code generation from the previous point, we can generate software that verifies whether or not the system is behaving as expected. If sufficient telemetry data is stored and we can link our semantic model with that data, the same software could also verify if the system behaved correctly during, for instance, the observations of the past night.

\subsection{An example model}

In the previous subsections, we have developed an ontology that defines so-called classes of things (e.g. formally: " $f_{s m}$ :State $r d f s:$ subClass0f owl:Thing"), and the properties between those classes. The fsm ontology does not define concrete instances of those classes, so essentially it only serves as a "meta-model" ontology for other (project-specific) "model" ontologies. In what follows, we will create such a "model" ontology for a simple experimental setup. The layout of this experiment is shown in Fig. 2: it consists of a motor, a legacy motor drive with a serial RS-485 interface, a PLC with a serial output module, and a piece of software representing a bi-stable switch. When the software switch is flipped, a serial command is sent to the drive, and the motor is forced to run at a given setpoint velocity.

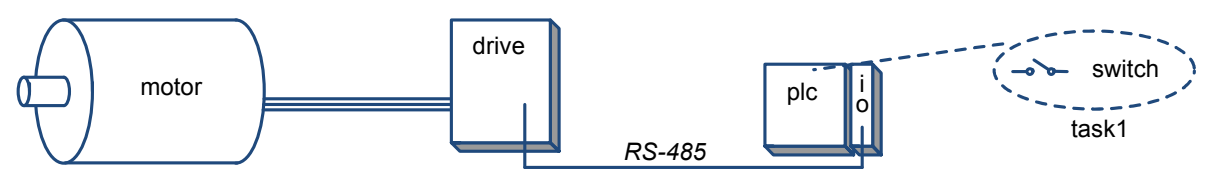

Figure 2. Example of an experimental setup used throughout the paper.

As explained in another paper, ${ }^{7}$ even simple experimental setups are hard to model in an ontology editor such as the one displayed in Fig. 1. Instead, we developed a software infrastructure that maps meta-model ontologies to Domain Specific Languages (DSLs). ${ }^{7}$ The fsm ontology for instance will be mapped to a DSL that has vocabulary terms such as fsm. State and fsm. hasRegion. Since the DSLs are based on CoffeeScript (an approach successfully demonstrated by other projects ${ }^{8}$ ), a web development tool or a simple text editor is sufficient to build models of setups like the one from our example. When the DSL scripts are executed, the contents of the DSL files will be converted to a standard Semantic Web format, which can be stored in an off-the-shelf knowledge base (see Fig. 3). An off-the-shelf template engine can now query the knowledge base using SPARQL, the de-facto query language for the Semantic Web, to generate source code and documentation. 


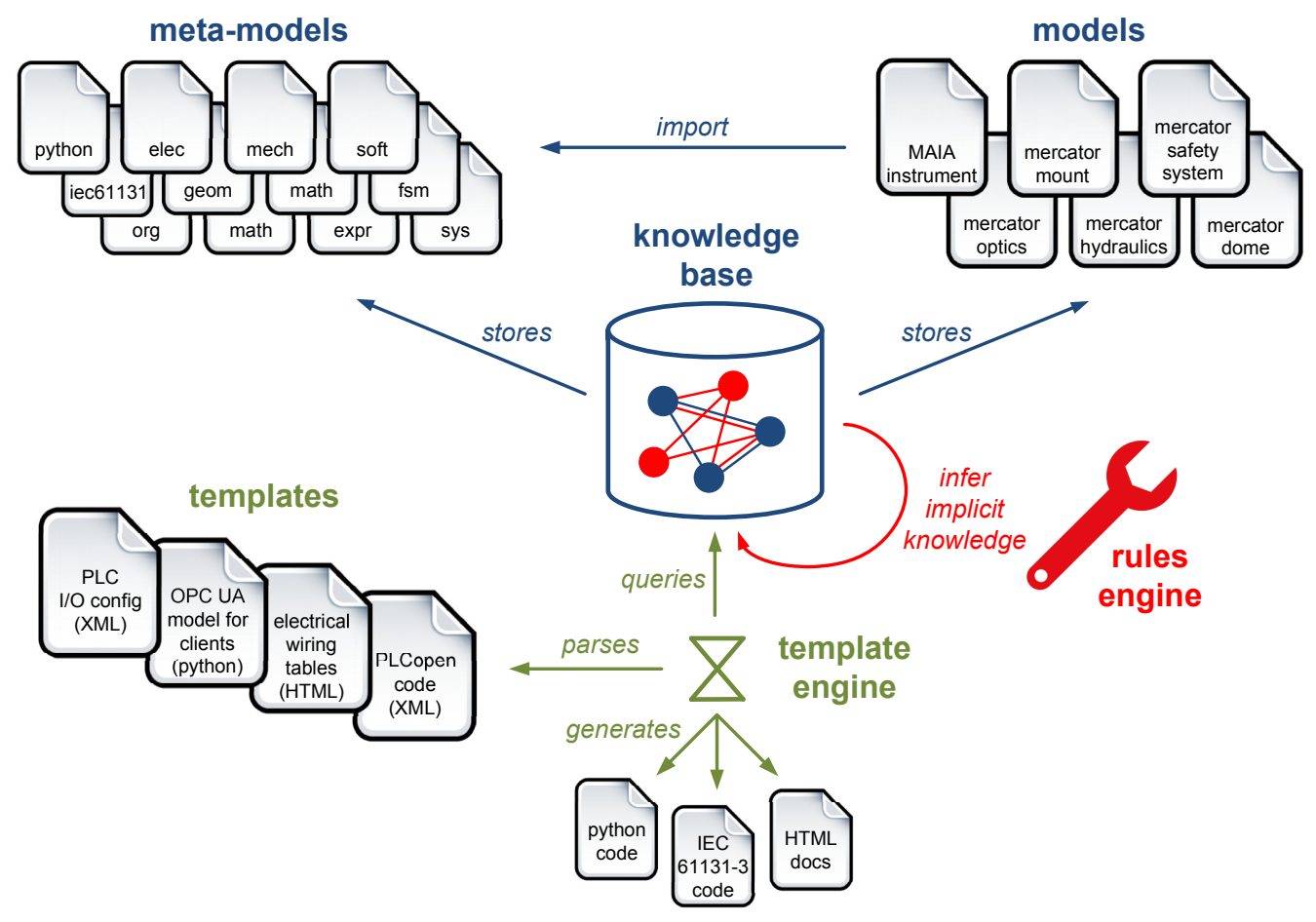

Figure 3. The workflow used throughout this paper.

To get an idea of the appearance of our DSL scripts, we have included a few code samples in Appendix A. The first sample shows a parametrized "macro" that can be used to model the sense of direction of a moving physical system (such as a motor), as an orthogonal region. This orthogonal region is a recurring pattern, captured by the DSL as a macro called MkDirectionRegion which we added to the csm or "common state machines" ontology. We can use this and other macros to model the motor type of a supplier called "X", as shown in Listing 3 . The $\mathrm{X}$.MkMotor_Y macro can finally be used to instantiate a motor within the ontology of our experimental setup: see Listing 4.

Note that the example provides some insight in how semantic models add "meaning" to the modeled entities. For instance, the velocity instance created by the X.MkMotor_Y macro represents a velocity not because we named it that way (which would be syntactic information), but instead because we modeled it as the velocity of a geometrical transformation between two mechanical parts (which is semantic information). A "smart" application could query a semantic model like this to learn for instance about all geometrical transformations between the rotating parts of a telescope drive system. Based on this information, the application could conclude that a motor speed of $1500 \mathrm{rpm}$ corresponds to a telescope speed of $3^{\circ} / \mathrm{sec}$, with both velocity vectors angled at $90^{\circ}$. Developing ontologies that can express this kind of knowledge is a challenging task, but our first experiments show that it is feasible with existing semantic web languages and rule languages such as SPIN.

\subsection{Summary}

In this section, we have demonstrated how control systems can be modeled using semantic languages. By combining meta-models (such as the fsm ontology) with project-specific models (such as the setupl ontology), we can create a true "knowledge base". The latter is essentially a database which stores not only all explicitly modeled information of our system, but also the information that is implicitly present. The implicit knowledge is the key to so-called "smart" applications: based on the explicit knowledge that we entered with our ontology editor and our DSL scripts, an off-the-shelf reasoning software or rules engine can generate much more knowledge about our modeled system. The result is a large knowledge base that can be analyzed for many different purposes such as troubleshooting, code generation, verification, etc. 


\section{IMPLEMENTATION}

\subsection{Semantic models vs. software models}

In the previous section, we have focused on the semantic models of physical objects such as electric motors. What we haven't explained yet is how the same semantic models can be used as the basis for software models. Our methodology is to develop both kinds of models simultaneously, and link them together (all within the DSL framework). This can be seen in Listing 4. The first 42 lines of code represent the semantic model of our experimental setup, according to the DSL code of Listing 2 and 3. In contrast, onwards from line 44 the DSL description is only about software modeling: it represents software objects as instances of soft:Instance, linked together by soft:hasAttribute relations. For each physical object on the first lines, there is a corresponding software instance on the last lines. Both are tied together via the soft:models relation.

In practice, it would take a lot of effort to map all properties of physical objects manually to attributes of software objects. Fortunately however, most of the knowledge needed to perform this mapping is already present in our semantic models. What's still needed is a formalism, i.e. a set of rules that unambiguously generate software models based on the semantics of physical object models. Such a rule is shown in Listing 1: it constructs a new software instance for each orthogonal region of a state machine. Similar rules can be created to construct software attributes for each state of an orthogonal region, for each condition of a state, etc. The generated software models are thus "implicit" knowledge of our models, as they are generated automatically by a rules engine. The result is one big coherent knowledge base that now also links the software properties to the other (electrical, mechanical, ...) properties of a control system.

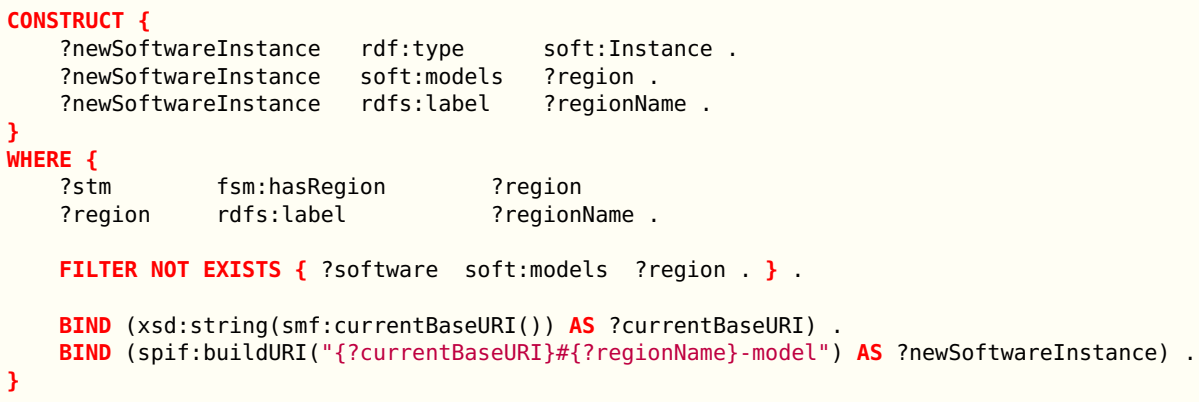

Listing 1. SPARQL query to construct a software instance for all orthogonal regions of a model.

\subsection{Software transformations}

The software model that was generated in the previous subsection talks in very general terms about software instances, types, attributes etc. This software model is often referred to as a PIM or Platform Independent Model. What's still missing is a transformation from this model to a PSM or Platform Specific Model that can be encoded by an actual programming language. In principle, any language that supports the concepts of our software ontology (e.g. soft:Instance, soft:Type, soft:Callable, soft:Attribute, ...) should be compatible. In practice we only focus on our main target languages: IEC 61131-3, or the set of standard languages to program PLCs. This set of languages has, in a sense, always been "object-oriented" because it defines stateful "function blocks" that can be instantiated. Since 2013 however, many more object-oriented extensions such as inheritance, polymorphism, methods, constructors, etc. have been introduced. Those object-oriented extensions give us a lot more freedom to find a "clean" transformation of the platform independent software models to IEC 61131-3 software code.

The code that will eventually be generated from the platform specific models, must meet a number of requirements. More specifically:

\section{Easy mixing of generated code with custom code.}

The code that will be generated from our models only represents the state machines that we have defined 
in our semantic model. Except for some "fundamental" behaviour (which can be modeled using causes and effects), no low-level behaviour is captured by our models. Therefore, the latter must be coded manually by a programmer, and "glued" to the generated state machine code.

\section{Predictable run-time behaviour.}

The cyclic operation of PLCs can lead to problems in distributed systems (see further). These problems must be dealt with correctly by our software.

\section{Optimal usage of OPC UA services and its support for information modeling.}

OPC UA provides a large number of services such as reading, writing, method calling, monitoring data changes and events, alarming, discovery, ... By carefully selecting the right service for the right use case, we can increase the efficiency and even the dependability of our control system. Furthermore, since both OPC UA and our DSLs are fundamentally semantic technologies, we can re-use the semantic models of our DSL scripts as OPC UA interfaces.

As we've learned, many of the intricacies of IEC 61131-3 (and PLC systems in general) come to the surface when trying to find a suitable PSM that meets the above requirements. Below we list some of the lessons that we've learned throughout the development process.

Lesson 1. The third edition of IEC 61131-3 facilitates a model-driven development approach.

In our model-driven architecture, we are generating code (based on the state machine models and fundamental behaviour), and glue that code together with custom developed code (that typically serves as low-level behavioural code). Several software patterns can accommodate this kind of "mixing". One of them is the "visitor pattern", which we've used in the past ${ }^{11}$ and which does not depend on object-oriented features that were absent in the original IEC 61131-3 standard. Now that object-oriented features have been added to the standard, we can apply the "template method pattern", ${ }^{9}$ which lets subclasses override methods that can be called by the superclass. In our case, the superclasses correspond to the generated code that represent the modeled state machines, orthogonal regions, states etc. The subclasses on the other hand must be coded manually by the developers who are responsible for the low-level behaviour.

The PSM representing the software switch of our example is shown in Fig. 4. We experimented with several other patterns too, but none of those attempts offered significant added value. For instance, interfaces (i.e. pure abstract classes) could force developers to implement certain behaviour of our state machines, but they exclude other patterns because non-pure abstract classes and multiple inheritance are not supported by IEC 61131-3.

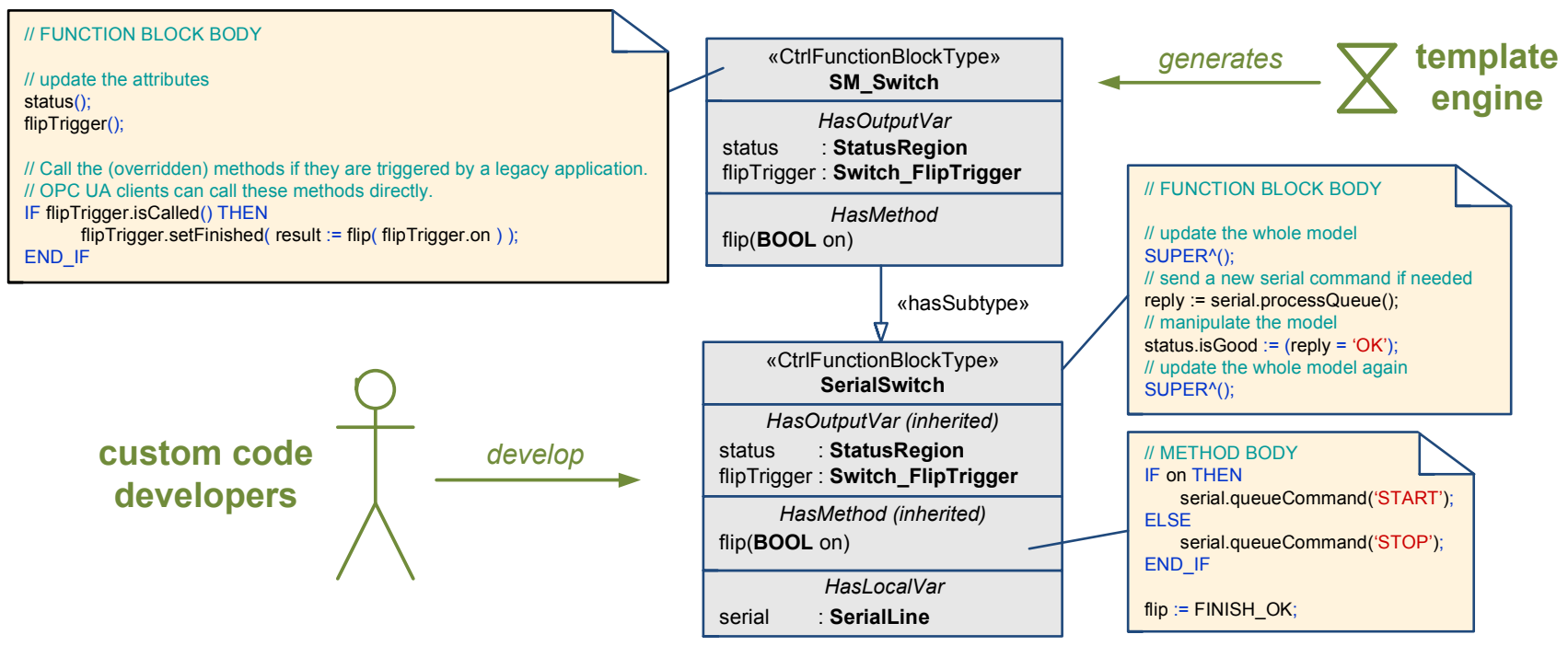

Figure 4. IEC 61131-3 model of the software switch of the example used throughout the paper. 
The approach of Fig. 4 turned out to be much more convenient than the approach based on the visitor pattern that we applied in earlier projects. The resulting code is much cleaner and its behaviour is easier to comprehend since the generated code and the custom code are tightly bound together. It is our goal to create a library (namespace) of frequently needed function blocks and corresponding user interface widgets, and re-use that library in several projects.

Lesson 2. OPC UA can extend a model-based development approach down to the plant floor level.

First of all, we'd like to emphasize that OPC UA is able to capture the same knowledge as our DSL scripts. OPC UA is essentially a semantic language, based on very similar principles as the RDF standard of the Semantic Web community. This means that any model we develop using the infrastructure of Fig. 3 can be represented "natively" by an OPC UA server. There's no need for a mapping or a conversion, i.e. both our DSL scripts and OPC UA interfaces can represent the same graph with the same nodes, the same URIs, the same links, ...

In case of PLCs, the OPC UA address space is not freely programmable. Instead, most PLC vendors support the PLCopen companion standard of OPC UA that defines a semantic model of the IEC 61131-3 standard. This companion standard is nothing more than a "meta-model" ontology that defines classes such as iec61131:CtrlFunctionBlockType and predicates such as iec61131:HasInputVars. Using this ontology, any PLC program loaded on a PLC can be exposed automatically by an OPC UA server. This means that the platform specific models of subsection 3.2 can be used not only for generating software code, but also for interfacing OPC UA clients and servers. Figure 4 thus represents not only the software running on the PLC, but also the OPC UA interface exposed by that PLC.

In previous experiments we had been developing graphical PLC software models and PIM-to-PSM transformations in a proprietary Computer Aided Software Engineering (CASE) tool. ${ }^{11}$ By adding an additional semantic layer (previously called "meta-model" ontologies) on top of the PIM, it becomes much easier to link our software models to other (electrical, mechanical, ...) engineering aspects of our project, and to existing ontologies such as the PLCopen one.

Lesson 3. Support for the more advanced parts of the OPC UA standard is growing ... but is still limited.

Compared to the "classic" OPC standard, OPC UA is still a very young technology with a comparatively small installed base. From the developers point of view, OPC UA is also more complex, even though third-party toolkits or our own open-source framework try to overcome this problem. ${ }^{12}$ All of this may explain why most commercial OPC UA servers and clients currently only support the features that were already offered by the former OPC standard: reading and writing variables, browsing an address space, and monitoring data changes. Alarms, events and historical data are additional services that a few vendors have already implemented in their products. In case of our state machine implementation, we can send events for instance whenever the active state of an orthogonal region changes.

What we've learned is that despite the efforts of some companies, there remains a big gap between what the OPC UA standard provides, and what is (commercially) available already right now. We feel that OPC UA has the potential to shift existing paradigms towards more decentralization and better (semantic) integration... but currently the implementation lags behind. One example is the support for OPC UA method calling on PLCs. As explained further on, this service can be a very "elegant" way to vertically integrate PLCs up to the level of Service Oriented Architectures (SOA). Even if the first supplier will soon release an OPC UA server that supports this feature, it will most likely take some time before SCADA (Supervisory Control And Data Acquisition) software companies provide support in their OPC UA clients. Another example is the OPC UA query service. Finding the right information in a complex address space appears to be a very valuable feature of a semantic technology such as OPC UA, yet we don't know any server that actually implemented this service.

Lesson 4. PLCs are no longer "closed" platforms due to standard exchange formats.

In previous applications, we have been generating code using a template engine that produced IEC 61131-3 compliant Structured Text (ST) output in plain text files. ${ }^{11}$ The programming environment of our PLC supplier could import those files, even though the exchange format was not standardized. In our new framework however we have migrated to a standard encoding format: we now export XML (eXtensible Markup Language) code that conforms to the schema published by the PLCopen organization. This XML schema is well documented and has 
become a mainstream exchange format supported by many suppliers in the automation industry. Since most of our generated code is structural (e.g. function block declarations), the produced XML code is uncomplicated. For the few lines of code that make up the implementation part of the function blocks, we generate structured text (ST) since text based languages are less cumbersome to generate than graphical languages. To our knowledge there is no standard XML schema yet of the third edition of IEC 61131-3, so currently a vendor-specific schema must be used instead for describing methods, inheritance, interfaces etc.

As the generated code is fully separated from the custom code, no language choice is imposed on the developers who provide the custom implementations. In practice however, we mostly rely on:

- Sequential Function Chart (SFC) to implement "slow" cyclic low-level behaviour (such as cyclic polling of serial devices);

- Structured Text (ST) to implement algorithms that do not involve the invocation of complex function blocks (such as mathematical algorithms);

- Ladder Diagram (LD) to implement boolean logic (which may involve the invocation of complex function blocks, such as motion control);

- Continuous Function Chart (CFC) to implement continuous processes (such as feedback control loops).

Lesson 5. PLCs can be deployed in service oriented architectures, if synchronization problems are dealt with.

A characteristic feature of PLCs is their cyclic operation, as shown in Fig. 5. It can be seen that the execution of the PLC program and the communication to external devices are separated in time, which can lead to unpredictable behaviour when multiple clients initiate the communication simultaneously. Consider a PLC program that increments an integer whenever a boolean variable is "true". What happens if the value of the trigger is written twice during the (very short) time span between two program executions? Will the integer be incremented twice, or will the PLC program never "notice" that the trigger has been updated twice? Another problem may occur when multiple variables need to be written consecutively. For instance, a motion control application may expose a "MoveAbsolute" function block instance that exposes variables to set the setpoint velocity, the setpoint acceleration and a trigger. What happens if two clients start to write these variables at the same time? Is it possible that the two requests are "mangled", so the motion is started with the wrong settings (e.g. the velocity setpoint of the first client and the acceleration setpoint of the second client)?

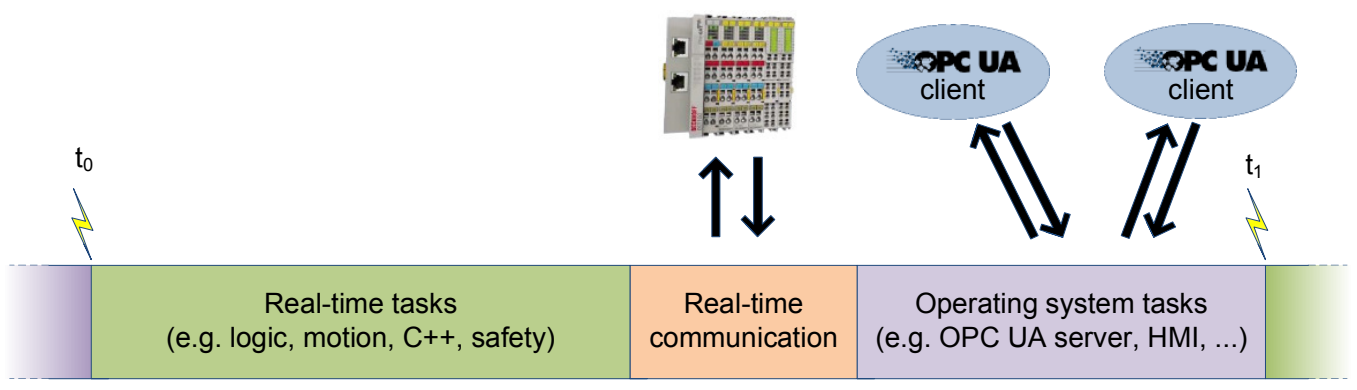

Figure 5. Cyclic operation of a soft-PLC.

The problems just described are essentially synchronization problems: several threads of execution require access to the same resources. In the IT world, problems like these are usually solved with mutexes. To our knowledge however, no such mechanism exists for our particular use case. A plausible explanation is that synchronization problems like these are very unlikely to occur in typical industrial PLC-based systems: cycle times are very small, the number of clients is very limited, and concurrent write requests to the PLC variables are very rare. Nevertheless, we strongly prefer to exclude all risk by using a recent innovation by our PLC supplier: the so-called "SOA PLC" or "Service Oriented Architecture PLC" by Beckhoff Automation. By exposing function block methods (a new object-oriented feature of IEC 61131-3) in the OPC UA address space of the PLC, remote 
clients can now invoke procedures directly on the PLC. ${ }^{10}$ We believe that this seemingly small technical feature is a very important one: in one call the client can now provide input to the server, the server can react on that input and can notify the client of the result. Since all communication requests are serialized (i.e. they are handled by a single thread of execution), the calls are atomic. This means that no synchronization problems can occur, and that PLCs can now safely be deployed in a service oriented architecture. Another advantage is that a single method call replaces several read and write requests between client and server, which increases efficiency. At the time of writing, the new feature was not commercially available yet but we were allowed to test a bèta version of the new software, and we hope to deploy a first application at our telescope site during the summer of 2014 .

\section{CONCLUSIONS}

In this paper we have presented a new methodology for developing state machine models that can be implemented on PLCs. By developing a meta-model for representing finite state machines based on Semantic Web technology, we have rooted our semantic models in formal logic. Thus, our state machine ontology allows us to formally specify the states, orthogonal regions, transitions etc. of our modeled systems, but also to reason about this knowledge and to infer more knowledge. This reasoning mechanism can be exploited to convert the state machine models into platform independent software models.

In the second part of the paper we show how those platform independent models can be mapped into platform specific models, again based on formalisms that we can define within our "ontology-based" software infrastructure. Our target platform is an IEC 61131-3 compliant soft-PLC that hosts an OPC UA server. Using a small experimental setup as an example, we demonstrate how the object-oriented features of IEC 611313 and companion standards of OPC UA facilitate a model-driven development approach. We also reflect on the availability and maturity of OPC UA implementations, and on the suitability of IEC 61131-3 w.r.t. code generation. Finally we identify the synchronization problems that may occur when PLCs are integrated in a service oriented architecture, and report a way to avoid those problems.

\section{REFERENCES}

[1] Kagermann, H., Wahlster, W. and Helbig, J., "Recommendations for implementing the strategic initiative INDUSTRIE 4.0 - Final report of the Industrie 4.0 Working Group," Acatech, München, 19-26 (2013).

[2] OPC Foundation, "OPC Unified Architecture, Pioneer of the 4th industrial (r)evolution," OPC Foundation, Scottsdale AZ, (2014).

[3] Allemang, D. and Hendler, J., [Semantic Web for the Working Ontologist], Morgan Kaufmann, Waltham MA, (2011).

[4] Smethurst, M., Styles, R. and Scott, T., "The Places Ontology," 27 November 2010, $<$ http://vocab.org/places/schema.html> (26 May 2014).

[5] Berners-Lee, T., Hendler, J., "The Semantic web," Scientific American Magazine, May 17 (2001).

[6] Knublauch, H., Hendler, A. J. and Idehen, K., "SPIN - W3C Member Submission," 22 February 2011, $<$ http://www.w3.org/Submission/spin-overview/> (26 May 2014).

[7] Pessemier, W., Raskin, G. Van Winckel, H., Deconinck, G. and Saey, P., "A practical approach to ontologyenabled control systems for astronomical instrumentation," Proc. ICALEPCS 2013, (2013).

[8] Filgueira, J. M., Bec, M., Soto, J., Lio, N. and Peng, C. Y.,"GMT software and controls overview," Proc. SPIE 8451, 845111 (2012).

[9] Gamma, E., Helm, R., Johnson, R. and Vlissides, J., [Design Patterns: Elements of Reusable Object-Oriented Software], Addison-Wesley, 325-344 (1995).

[10] Hoppe, S., "SOA enabled PLC: Remote Service execution into the IEC61131-3 controller," OPC Day Europe 2014, Esslingen, (14 May 2014).

[11] Pessemier, W., Deconinck, G., Raskin, G., Saey, P. and Van Winckel, H., "Design and First Commissioning Results of PLC-based Control Systems at the Mercator Telescope," Proc. SPIE 8451, 84512V (2012).

[12] Pessemier, W., Deconinck, G., Raskin, G., Saey, P. and Van Winckel, H., "UAF: a generic OPC Unified Architecture Framework," Proc. SPIE 8451, 84510P (2012). 


\section{APPENDIX A. EXAMPLE}

\section{models/common/statemachines. coffee (excerpt)}

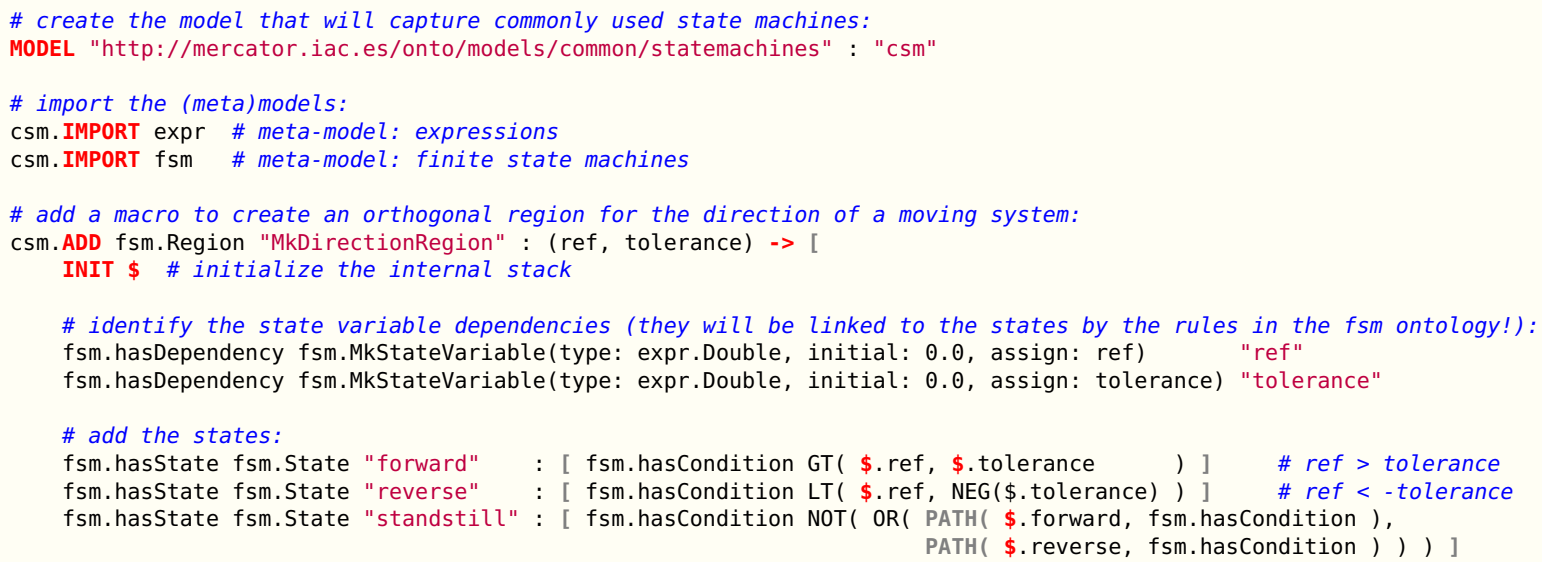

Listing 2. A DSL macro to create an orthogonal region.

\section{models/suppliers/x. coffee (excerpt)}

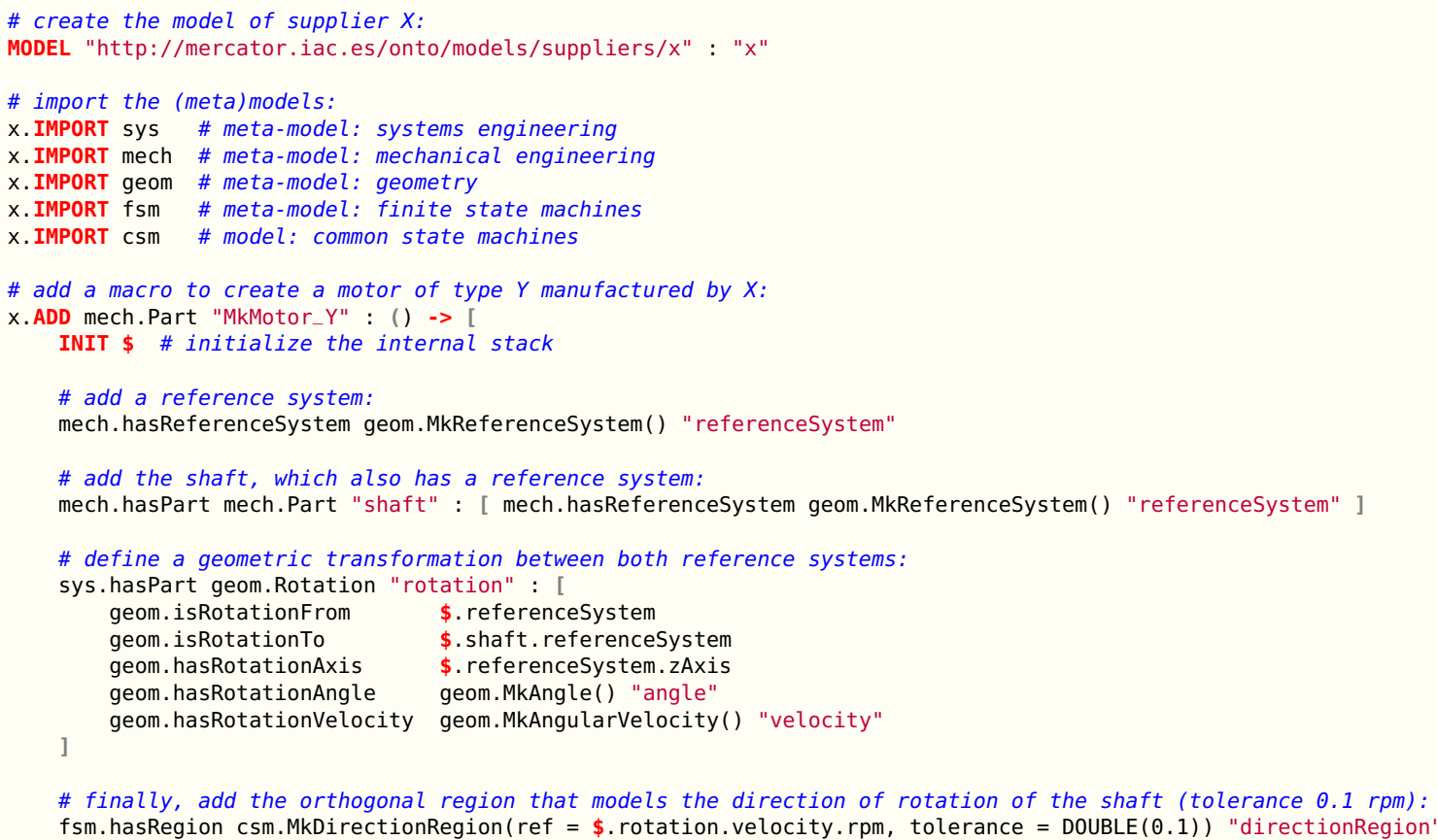

Listing 3. A DSL macro to create a motor of manufacturer X. 


\section{models/experiments/setup1. coffee (excerpt)}

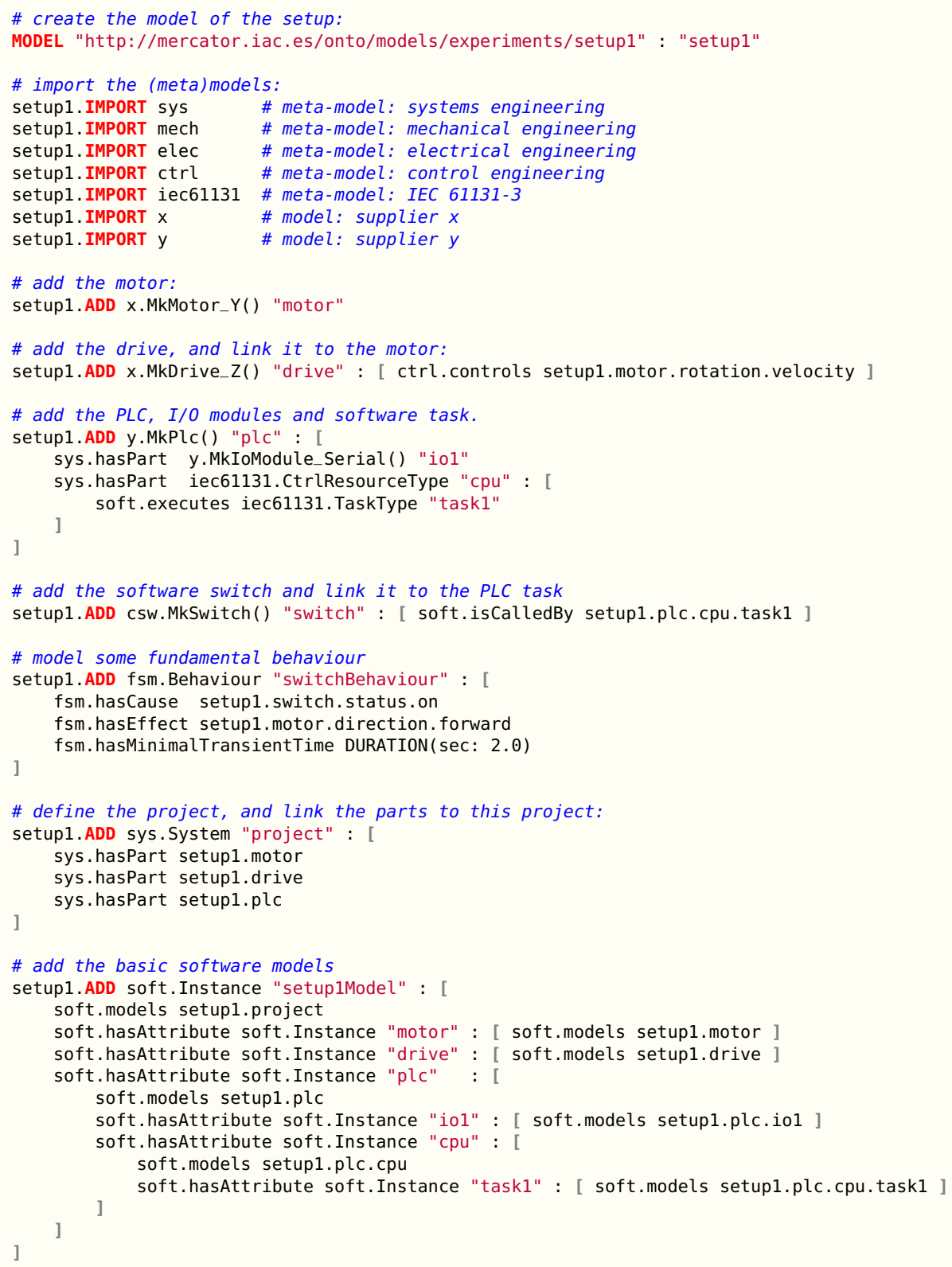

Listing 4. The final model of our experimental setup. 